\title{
Hand-Eye Calibration of a Robot - UltraSound Probe System without any 3D Localizers
}

\author{
Johan Sarrazin $^{1,2}$, Emmanuel Promayon ${ }^{2}$, Michael Baumann ${ }^{1}$ and Jocelyne Troccaz ${ }^{2}$
}

\begin{abstract}
D UltraSound (US) probes are used in clinical applications for their ease of use and ability to obtain intraoperative volumes. In surgical navigation applications a calibration step is needed to localize the probe in a general coordinate system. This paper presents a new hand-eye calibration method using directly the kinematic model of a robot and US volume registration data that does not require any 3D localizers. First results show a targeting error of $2.34 \mathrm{~mm}$ on an experimental setup using manual segmentation of five beads in ten US volumes.
\end{abstract}

\section{INTRODUCTION}

Today, 3D US volumes are increasingly used in clinical applications. The trade-off between the ease of use, the convenience, the image quality and the acquisition time of 3D volumes facilitate the development of new US guidance techniques in medicine. During medical procedures intraoperative US volumes can be used to register other intraoperative US volumes or to guide the surgeon during interventions. These US volumes can also be registered with preoperative MR or CT images where the definition of targets can bring valuable information.

However, the US probe alone does not provide any way of positioning each US volume in a single reference frame. In a majority of medical applications, the calibration of the probe is needed to register all data in the same mathematical space. To overcome this shortcoming the literature mainly presents two types of calibration techniques using external 3D localizers [1]. In these techniques, a tracking reference frame is fixed on the probe and US volumes are acquired from a tracked phantom immersed in a water bath, as it explained in [1], [2] and [3], or by directly registering US volumes [4].

During ultrasound guided prostate biopsy intervention, a well-distributed tissue sampling has to be performed using TransRectal UltraSound volumes (TRUS) in order to assess the presence of prostate cancer. A robot described in [5] that holds the 3D US probe may be used to efficiently sample areas in the prostate. The calibration of this system could be an effective way to collect additional data to enable registrations. The calibration methods describe above have the drawbacks of requiring an external equipment such as tracking device and calibration rig. The reference frame fixed

\footnotetext{
*This work is supported by Koelis, ANRT and ANR grants (PROSBOT project ANR-11-TECS-0017 and CAMI labex ANR-11-LBX-0004)

${ }^{1}$ KOELIS SAS, 5. av du Grand Sablon, La Tronche, F-38700, France. nameekoelis.com

${ }^{2}$ Univ. Grenoble Alpes, TIMC-IMAG, F-38000 Grenoble, France. CNRS, TIMC-IMAG, F-38000 Grenoble, France. firstname. namedimag. fr
}

on the probe has to be detected by the tracking device which generally comes with a loss of movements' freedom. The use of large water basin or any other bulky device may also be problematic for day to day clinical use or involve additional electrical risks.

From these observations, a new method to calibrate a robot - US probe holder system by using the robot kinematic model and a 3D US volume registration algorithm was developed. Probe positions and orientations can be calibrated to the US volumes. In a previous work [6], a feasibility study of this method was performed. Preliminary results were obtained by simulating the robot via an optical Polaris localizer (NDINorthern Digital Inc., Canada) and a tracker reference frame rigidly fixed on the 3D US probe. This initial study gave us a RMS targeting error of $1.83 \mathrm{~mm}$. It also allowed us to obtain a test bench to evaluate the accuracy of the robot compared to the existing tracking methods using optical localizers. Another interesting finding was that the manual segmentation of glass beads, serving as fiducials, in US volumes of average quality may induce a significant noise in the calibration result.

In this paper, we present the first results obtained by this hand-eye calibration method using the actual robot on a real experiment setup with a phantom. We evaluated the robustness of the method by performing a sensitivity study based on the impact of the segmentation noise on one hand, and the impact of the noise induced by both the robot sensors and by the registration method on the other hand. To describe this work, the method is presented in Section II. In the third section, the targeting error resulting during the experimental setup using the robot and the sensitivity study based on simulated data are detailed and discussed. Finally, concluding remarks are provided in the last section.

\section{METHOD}

The method presented in this paper adapts a standard handeye calibration algorithm [7], [8] to estimate the rigid transformation $X$ from US volume frames to the robot coordinate system. A phantom is used to acquire all the necessary US volumes and robot positions. As it is represented in Fig.1 we respectively use an US volume registration algorithm and the kinematic model of the robot to obtain the $A_{i}$ and $B_{i}$ matrices. $A_{i}$ are the transformations from the current 3D US volume to a reference US volume acquired at the beginning of the experiment. $B_{i}$ are the transformations from the current 3D US probe position to the robot coordinate system. The calibration matrix $X$ is then obtained by solving 
the problem $A X=X B$ with a minimum number of three measures with different rotations and translations.

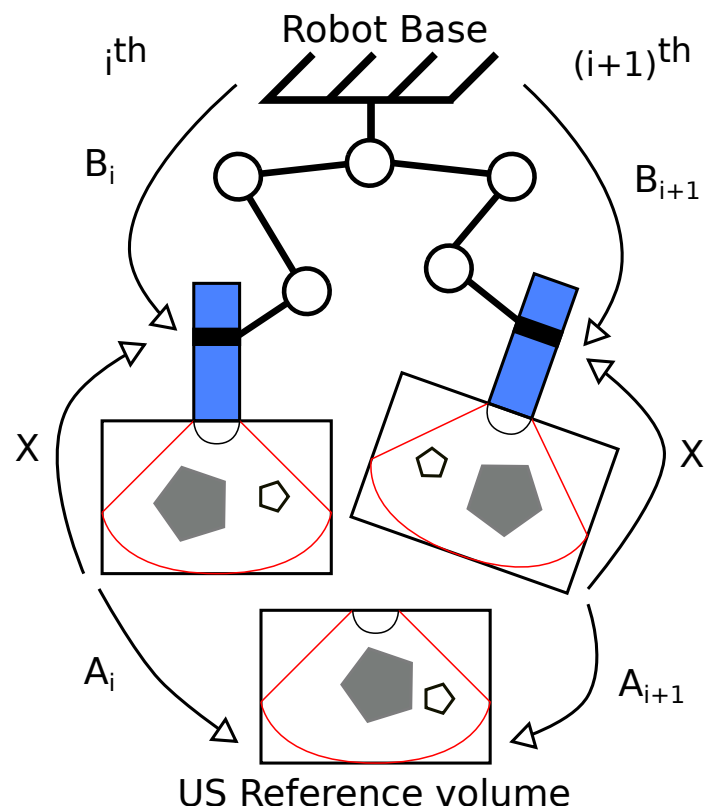

Fig. 1. Application of the hand-eye calibration problem with a robot probe holder and a 3D US probe.

\section{A. Replacing the $3 D$ localizers systems}

In the standard calibration methods found in the literature a tracking system is used to obtain the position of the US probe in the localizer reference frame. Rigid trackers (usually called rigid bodies) are fixed on the 3D US probe. The rigid bodies provide the $B_{i}$ transformations (Fig.1). Some methods track the phantom using another rigid body to obtain the transformation between the localizer and the phantom, i.e the $A_{i}$ transformation (Fig.1). If the phantom is not tracked itself, then a registration method is used to register the US volumes between each other. Our method does not require any additional 3D localizer by using:

- a 3D US registration method,

- a phantom specifically designed for the calibration,

- the kinematic model of a robot.

The 3D US volume registration was used to compute the $A_{i}$ transformations. This registration method is provided by the Urostation (Koelis, La Tronche, France). It is the same algorithm used during TRUS biopsy session. The registration accuracy of this application is $0.8 \mathrm{~mm}$.

A phantom made up of PolyVinyl Chloride (PVC) [9] was built with a prostate casting from a segmented MRI, see Fig. 2 a). Its size is $16 \mathrm{~cm} \times 12 \mathrm{~cm} \times 9 \mathrm{~cm}$. The phantom was built with a target Young Modulus of $150 \mathrm{kPa}$. For this experiments the same phantom is used for the calibration and the acquisitions. As a result, the speed of sound in PVC may not be taken into account for this evaluation. A series of clay beads with a diameter smaller than $2 \mathrm{~mm}$ were added in the phantom in order to compute a targeting error after the calibration process (section II-B).
The kinematic model of a robot, developed by [5], was used to obtain the $B_{i}$ transformations. The use of the robot instead of a $3 \mathrm{D}$ localizer is possible because the $3 \mathrm{D}$ US probe is rigidly fixed to the robot arm. The rotations and the translations of the probe are therefore taken into account in the robot's kinematic model. A reference position (orientation and translation) of the probe can be computed at any time in the robot coordinate system, which then can be used to solve the hand-eye problem.

\section{B. Measuring the calibration error}

To measure an error in the calibration process, the properties of our PVC phantom are used. The beads are manually segmented in the US volumes, Fig. 2 b), and then projected in the robot coordinate system thanks to the $X$ matrix obtained by the hand-eye calibration and the $B_{i}$ matrices measured during the acquisitions. The Root Mean Square (RMS) targeting error is then computed as the distance of each projected bead to the mean position in the robot frame:

$$
\epsilon_{R M S}=\sqrt{\frac{\sum_{i=1}^{n} \sum_{j=1}^{m}\left(Q_{R}^{j}-\hat{P}_{i R}^{j}\right)^{2}}{n . m}}
$$

where $Q_{R}^{j}=\frac{1}{n} \sum_{i=1}^{n} \hat{P}_{i R}^{j}$ is the mean position of the $j^{t h}$ bead in the robot frame and $\hat{P}_{i R}^{j}$ is the estimated position of the $j^{\text {th }}$ bead in the $i^{\text {th }}$ US volumes transformed to the robot coordinate system. Note that $\hat{P}_{i R}^{j}=B_{i} \cdot X^{-1} \cdot \hat{P}_{i U S}^{j}$, where $\hat{P}_{i U S}^{j}$ is the position of the bead $j$ manually segmented in the US volume frame $i$.
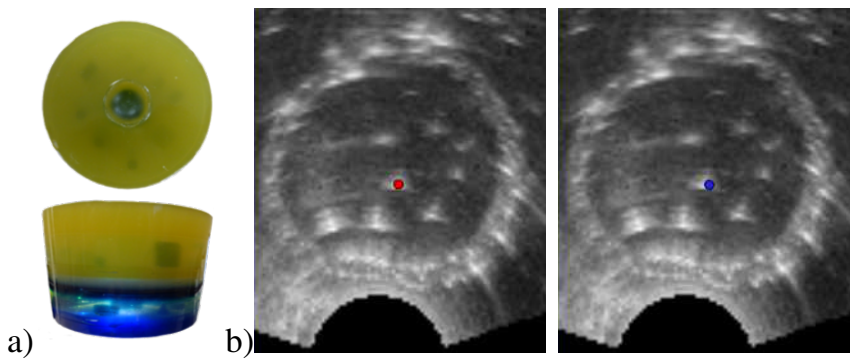

Fig. 2. (a) The specifically designed phantom contains glass beads and a look-alike prostate. (b) Beads and prostate are visible in the US images and can be manually segmented.

\section{Generation of virtual bench tests}

To perform the sensitivity study, we developed a specific tool in the CamiTK framework [10] to generate synthetic data sets and add some noise to them. Virtual reference frames symbolizing respectively the robot and the reference US volume are randomly generated. Then, a virtual tool composed of two reference frames, where the generated $\mathrm{X}$ transformation between these two coordinate systems is known, is displaced randomly in the scene. Each frame of the tool is computed in the virtual robot frame and in the virtual reference volume frame to obtain the $A_{i}$ and the $B_{i}$ matrices. A combination of Gaussian or Uniform noise can be added randomly to these virtual acquisitions in rotations and/or translations. Finally we solved the hand-eye problem 
to obtain the calibration matrix $X$ and observe the impact of the noise on this matrix. This method mimics the experiments with the actual robot in terms of rotations and translations. To study the impact of the manual segmentation step, nine points are generated in the reference US volumes and computed in each new US volume. To have realistic data set, these points represent a cube and its centroid of a size similar to a classical TRUS volume. A Gaussian noise is added to these points to study the impact of the segmentation noise.

\section{EXPERIMENTS}

On the basis of the previous feasibility study, we decided to evaluate the calibration method with both synthetic and real data sets to respectively observe the impact of the noise in the calibration process and the ability of the robot to precisely track the probe movements. Each subsection presents the experimental setup, the results and the related discussion.

\section{A. Impact of the segmentation}

To study the impact of the segmentation error on $\epsilon_{R M S} 12$ virtual acquisitions were generated. Gaussian noise is added progressively to the synthetic point coordinates from $\sigma=0$ to $\sigma=1$ by step of 0.1 . Fig. 3 presents the variation of $\epsilon_{R M S}$ in function of the additional noise.

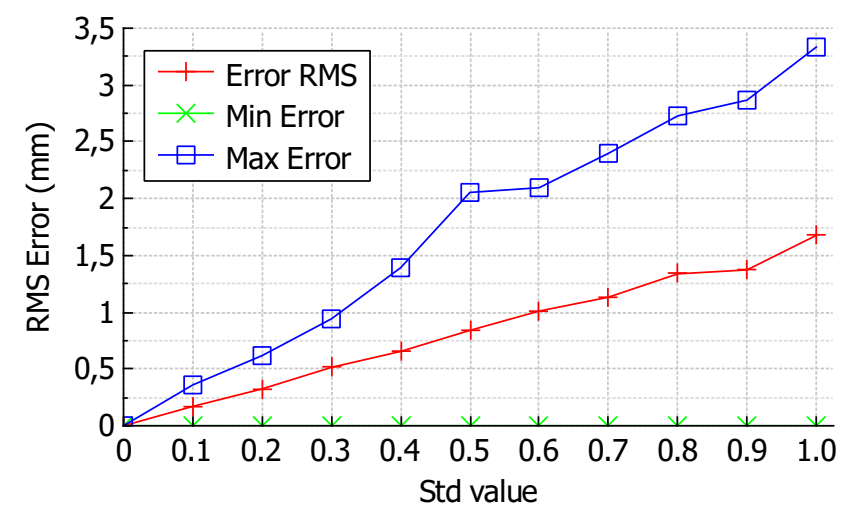

Fig. 3. $\epsilon_{R M S}$ in function of the segmentation additional noise.

The graph clearly shows that the quality of segmentation impacts the final calibration error. In our previous study, we observed that the error due to the manual segmentation was ranged from 1 to 1.5 voxels, where voxel size was $0.57 \mathrm{~mm} \mathrm{x}$ $0.57 \mathrm{~mm} \times 0.57 \mathrm{~mm}$. The maximum deviation error of a manually segmented bead was consequently between $0.99 \mathrm{~mm}$ and $1.48 \mathrm{~mm}$. Fig. 3 shows that such a segmentation error is equivalent to a centered gaussian noise ranged from $0.5 \mathrm{~mm}$ to $0.9 \mathrm{~mm}$ STD in the final $\epsilon_{R M S}$.

If we crosscheck these results with our previous calibration results, the error induced by the segmentation can stand for up to $50 \%$ of the total $\epsilon_{R M S}$. It also means that with a perfect calibration the accuracy of the clinical application is highly dependent on the US volumes and segmentation quality.

\section{B. Impact of the kinematic model and the image registration}

To study the impact of the kinematic model and the image registration on $\epsilon_{R M S} 150$ set of 20 virtual acquisitions were generated with a translation motion range of $[-5 ; 5] \mathrm{cm}$ and a rotation range of $[0 ; 60]^{\circ}$. Gaussian noise is added progressively to the 150 sets from $\sigma=0$ to $\sigma=1$ by step of 0.1 , generating in total 33,000 acquisitions, each including a couple of noisy $A_{i}$ and $B_{i}$. The noise mimics the volume registration noise in the $A_{i}$ transformations and the noise induced by the robot sensors in the $B_{i}$ transformations. For each of the 1,650 sets, $\epsilon_{R M S}$ was computed depending on the number of acquisitions used in the hand-eye algorithm, using $4,8,12,16$ or 20 acquisitions. Fig.4 presents the root mean square of the $\epsilon_{R M S}$ errors in function of the additional noise level and the number of acquisition used for the calibration in the case of an exact segmentation.

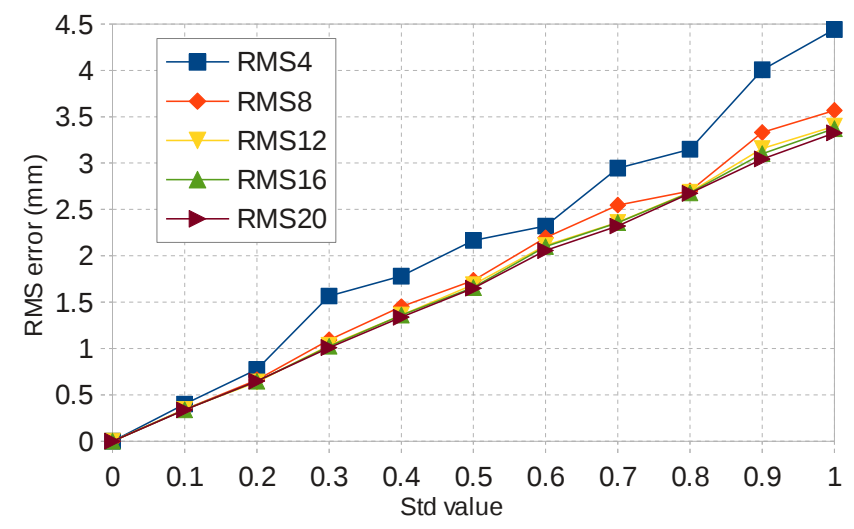

Fig. 4. Evolution of the calibration error when Gaussian noise is gradually added to the $A_{i}$ and the $B_{i}$ transformations.

The graph shows that the noise in $A_{i}$ and $B_{i}$ transformations impacts the calibration results and that $\epsilon_{R M S}$ also depends on the number of acquisitions used to solve the $A X=X B$ system. Although using only 4 acquisitions for the calibration step would be faster in a medical application, the resulting error would be high. While using 8 acquisitions seems better, it shows more noise sensitivity with some local peaks. Using 12 or more acquisitions does not change significantly the resulting error. The difference range in $\epsilon_{R M S}$ for the calibration performed with 12,16 and 20 acquisitions is less than $0.12 \mathrm{~mm}$.

Twelve acquisitions seem to be a good compromise to obtain a robust and accurate result while minimizing the acquisition time required by this step.

\section{Robot Experiments}

For the first experiments using the robot probe holder and the dedicated phantom a series of 14 acquisitions were made, that includes 14 kinematic states of the robot and 14 US volumes. Acquisitions 1 to 12 were used to solve the $A X=$ $X B$ problem. Five beads were then manually segmented in eight US volumes used to solve the hand-eye algorithm (i.e., v1 to v8) in order to evaluate the accuracy of the process. Two other US volumes independent of the calibration process 
were also used to assess the robustness of the calibration (i.e., v9 and v10). Fig.5 shows $\epsilon_{R M S}$ for each segmented beads for volumes $v_{1}$ to $v_{10}$.

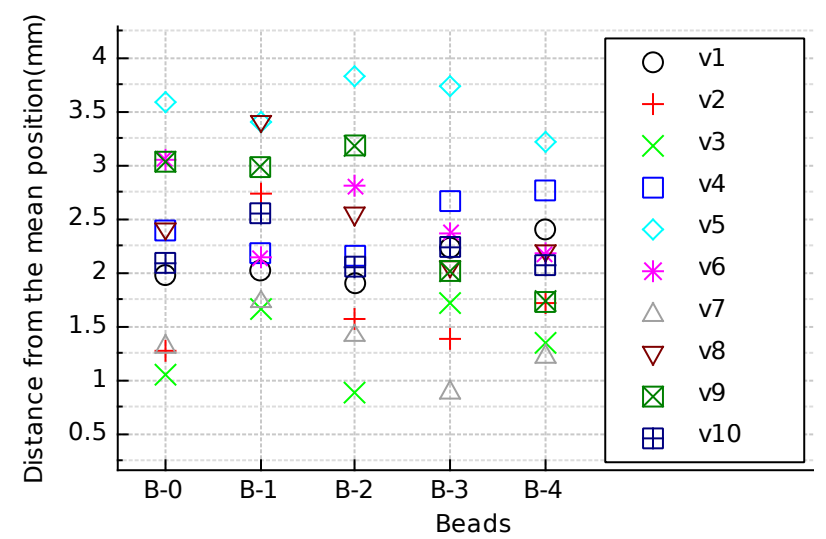

Fig. 5. $\epsilon_{R M S}$ for each segmented beads for volumes $v_{1}$ to $v_{10}$.

TABLE I

$\epsilon_{R M S}$ FOR FIVE SEGMENTED BEADS.

\begin{tabular}{|l|c|c|c|c|}
\hline & RMS & Min & Max & Std \\
\hline All US volumes & 2.34 & 0.87 & 3.82 & 0.73 \\
\hline Without v5 US volume & 2.12 & 0.84 & 3.31 & 0.61 \\
\hline
\end{tabular}

$\epsilon_{R M S}$ for this experiment is $2.34 \mathrm{~mm}$ with a standard deviation of $0.73 \mathrm{~mm}$, see Table I. Fig 5 shows that the maximum targeting error is given by the US volume $v_{5}$. The errors can come from the registration step, the segmentation step or can be dependent to the experimental setup (for instance it can be due to the physical constraints applied to the end-effector of the robot). If we consider volume $v_{5}$ as an outlier, $\epsilon_{R M S}$ can notably be improved to $2.12 \mathrm{~mm}$.

Compared to the literature, our results of $2.34 \mathrm{~mm}$ with the targeting point error are very encouraging. [1], [11] and [4] respectively found a RMS targeting point error of $3.5 \mathrm{~mm}$ by using $12 \mathrm{2D}$ images, $2.37 \mathrm{~mm}$ with 56 US volumes and $2.0 \mathrm{~mm}$ with $36 \mathrm{US}$ volumes. These hand-eye methods use accurate 3D localizers to obtain $B_{i}$ matrices and registration tools to obtain $A_{i}$ matrices. The comparison of these results with our sensitivity study shows that the $\epsilon_{R M S}$ can rise sharply with a rotation and a translation noise (lesser than respectively $\sigma=0.5^{\circ}$ and $\sigma=0.5 \mathrm{~mm}$ ) induced by the robot sensors and by the registration tool. Compared to our previous work, the use of a robot instead of a 3D localizer generates an error of approximately $0.5 \mathrm{~mm}$. This error might mainly be induced by the use of the robot sensors but it is acceptable compared to the calibration results using a tracker device found in the literature.

On the basis of these results, other paths can be explored to improve our method such as the design of a better and more echogenic phantom to have the possibility to segment automatically shapes and points and to improve the registration. During medical interventions using a 3D US probe, the number of acquisitions is limited. As a consequence, the hand-eye calibration problem cannot be solved with a high number of positions to have a robust $X$ matrix. Rejection criteria algorithms could so be implemented to eliminate acquisitions considered as outliers due to a bad registration [4] or induced by a noise in the robot.

\section{CONCLUSIONS}

We have proposed the first results of a new method to calibrate an ultrasound probe held by a robot by using the robot kinematic model and the image registrations as inputs of a hand-eye calibration problem. Unlike most of ultrasound probe calibration methods found in the literature, our method does not require any additional 3D localizer. Despite the use of less accurate systems compared to tracking devices, the resulting precision is good. Generated and real experiments have been performed to evaluate the performance of our method. Numerically, the calibration with 12 acquisitions seems to be enough as shown in our experiments. Finally, the trade-off between the ease of use of the method and the resulting RMS targeting error of $2.34 \mathrm{~mm}$ is very encouraging.

\section{ACKNOWLEDGMENTS}

The authors would like to thank M.A. Vitrani, P. Mozer, and D. Reversat for their technical and logistic support to use the robot as well as S. Voros and A. Agustinos for their help on the hand-eye calibration.

\section{REFERENCES}

[1] C. Bergmeir, M. Seitel, C. Franck, R. De Simone, H. P. Meinzer, and I. Wolf, Comparing calibration approaches for 3D ultrasound probes. International journal of computer assisted radiology and Surgery,vol. 4, 2009, pp. 203-213

[2] M. Baumann, V. Daanen, A. Leroy, and J. Troccaz, 3-D Ultrasound probe calibration for computer-guided diagnosis and therapy. Proceedings of Computer Vision Approaches to Medical Image Analysis, Second International Workshop, 2006 (CVAMIA'06), pp. 248-259.

[3] R. W. Prager, R. N. Rohling, A. H. Gee, and L. Berman, Rapid Calibration for 3-D Freehand Ultrasound. Ultrasound in Medicine and Biology, vol. 24, 1998, pp. 855-869.

[4] J. Schlosser, C. Kirmizibayrak, V. Shamdasani, S. Metz, D. Hristov, Automatic 3D ultrasound calibration for image guided therapy using intramodality image registration. Physics in Medicine and Biology, vol. 58, 2013, pp. 7481-7496.

[5] C. Poquet, P. Mozer, M. A. Vitrani, G. Morel, An endorectal ultrasound probe comanipulator with hybrid actuation combining brakes and motors. Mechatronics, IEEE/ASME Transactions on, 1-11, 2014.

[6] J. Sarrazin, E. Promayon, M. Baumann, J. Troccaz, 3D Ultrasound probe calibration using robotic arm and image registration. Surgetica' 14, 2014.

[7] R. Y. Tsai, and R. K. Lenz, A new technique for fully autonomous and efficient 3D robotics hand/eye calibration. IEEE transactions on Robotics and Automation, vol. 5, 1989, pp. 345-358.

[8] Z. Zhao, Hand-eye calibration using convex optimization. Robotics and Automation (ICRA), 2011 IEEE Int. Conf., pp. 2947-2952.

[9] N. Hungr, J. A. Long, V. Beix, and J. Troccaz, A realistic deformable prostate phantom for multimodal imaging and needle-insertion procedures. Medical Physics, vol. 39, no. 4, 2012, pp. 2031-2041.

[10] E. Promayon, C. Fouard, M. Bailet, A. Deram, G. Fiard, N. Hungr, V. Luboz, Y. Payan, J. Sarrazin, N. Saubat, S. Yuki-Selmi, S. Voros, P. Cinquin, J. Troccaz, Using CamiTK for rapid prototyping of interactive Computer Assisted Medical Intervention applications. IEEE Engineering in Medicine and Biology Conference (EBMC), 2013, pp. 4933-4936.

[11] X. Huang, L. F. Gutierrez, D. Stanton, P. C. Kim, and A. Jain, Image registration based 3D TEE-EM calibration. Biomedical Imaging: From Nano to Macro, IEEE International Symposium on, 2010, pp. 12091212. 